\title{
Caracterização de oito raças do bicho-da-seda (Bombyx mori L.)
}

\author{
Characterization of eight silkworm races (Bombyx mori L.)
}

\author{
Antonio José Porto ${ }^{1}$ Fumiko Okamoto $^{1}$ Eduardo Antonio da Cunha $^{2}$ Ivani Pozar Otsuk $^{3}$
}

RESUMO

$O$ experimento foi conduzido na Estação Experimental de Zootecnia de Gália, do Instituto de Zootecnia, $S A A-S P$, no ano de 2000. Oito raças de bicho-da-seda, de origem Japonesa e Chinesa foram estudadas (B101, B102, B104, B109, C201, C202, C203, C208) em relação a caracteres biológicos (Ganho de peso total de uma lagartaGP, Porcentagem de mortalidade-MO, Número de machos$N M$, Número de fêmeas-NF, Número de ovos/postura-OP e Porcentagem de eclosão-EC) e caracteres de produção de casulo (Peso unitário da glândula sericígena-GS, Peso de 30 cascas séricas-CS, Peso de 30 crisálidas-PC, Teor de seda líquido-TS, Casulos desclassificados-CD, Comprimento do casulo-CC e Largura do casulo-LC). O delineamento experimental foi o inteiramente casualizado, com quatro repetições/raça. Não houve variação entre as raças para $G P$, MO, NF e OP. A raça B101 apresentou, no geral, um menor NM e uma menor EC. Quanto à produção de casulos, no geral, os melhores resultados foram apresentados pela raça C202, com um bom GS (38\% do peso final da lagarta), um dos mais altos CS e TS e valores próximos da média para PC, $C D, C C$ e LC. A raça C201, em relação ao casulo produzido, apresentou os piores resultados.

Palavras-chave: caracteres biológicos, casulo, raça.

\section{ABSTRACT}

The experiment was developed at Estação Experimental de Zootecnia de Galia - Instituto de Zootecnia, SAA-SP, Gália city, São Paulo, Brazil, on 2000. Eight silkworm races of Japanese and Chinese origin were studied (B101, B102, B104, B109, C201, C202, C203, C208) for biological characters ( Total weight-gain for one caterpillar$G P$, Percentage of mortality-MO, Number of male-NM, Number of female-NF, Number of egg/laying-OP and Percentage of eclodibility-EC) and for characters of cocoon production (silk gland unitary weight-GS, 30 cocoon shell weight-CS, 30 chrysalis weight-PC, silk net purport -TS, disqualified cocoon-
$C D$, cocoon length-CC and cocoon breadth $-L C)$. It was used a completely randomized design, with four replications/ race. It was not detected variation among races for GP, MO, NF and OP. The B101 race showed a smaller NM and a smaller $E C$. With regard to cocoon production, the C202 race showed the best result, with good GS (38\% of the final weight of the caterpillar), one of highest CS and TS and values next to the average for $P C, C D, C C$ e $L C$. The C201 race, with regard to cocoon produced, showed the worst results.

Key words: biological characters, cocoon, race.

\section{INTRODUÇÃO}

O bicho-da-seda, Bombyx mori L. (Lepidoptera : Bombycidae) (PANG-CHUAN e DACHUANG, 1992), é originário do Bombyx mandarina (Theophila mandarina), que apresenta características morfológicas semelhantes e capacidade de acasalamento (YOKOYAMA, 1959).

De acordo com a distribuição geográfica, o bicho-da-seda pode ser ainda classificado e identificado como de origem Japonesa, Chinesa, Europeia ou Indiana (KRISHNASWAMI et al., 1979). OKINO (1982) descreveu variações nas características do ovo (cor, forma), da lagarta (resistência, tamanho, cor, número de gerações/ano, número de ecdises) e do casulo (forma, tamanho, cor, rendimento) do bichoda-seda, em função de sua origem.

Os atributos qualitativos e quantitativos, intrínsicos de cada raça ou linhagens, são normalmente estudados (ABREU et al., 1960a; RAVINDRA et al., 1990; RAJU \& KRISHNAMURTHY, 1993), visando selecionar os melhores para programas de

${ }^{1}$ Pesquisador Científico da Estação Experimental de Zootecnia de Gália, Instituto de Zootecnia, SAA-SP, CP 16, 17450 000, Gália, SP, Brasil. E-mail: updgalia@ig.com.br. Autor para correspondência.

${ }^{2}$ Pesquisador Científico do Centro de Etologia, Ambiência e Manejo, Instituto de Zootecnia, SP.

${ }^{3}$ Pesquisador Científico do Centro de Métodos Quantitativos, Instituto de Zootecnia-SP. 
melhoramento e formação de híbridos. O primeiro passo para iniciar um programa de melhoramento de qualquer espécie animal ou vegetal é o levantamento da variabilidade disponível. Segundo FONSECA \& FONSECA (1988), quando se procura desenvolver um trabalho de melhoramento do bicho-da-seda, torna-se necessário um levantamento de todos os caracteres de importância sericícola, relacionados com a natureza do ovo, da lagarta, do casulo e da mariposa, podendo ser levantadas mais de 20 variáveis.

Conforme BRASLAVSKY et al. (1997), além do índice de sobrevivência da lagarta, considerado como fator chave em todo programa de seleção, do qual depende a produção do casulo, outras características quantitativas (teor de seda, conteúdo de fibra na casca sérica, comprimento, rendimento e uniformidade da fibra) desempenham variado papel em diferentes programas, conforme o objetivo procurado e são fundamentais quando se criam raças especializadas.

Em muitos casos, somente esse levantamento é suficiente para se obter o avanço desejado e, em decorrência dessa operação, é necessário formar um banco de germoplasma que contenha todo material coletado de maneira organizada com o máximo de informações possíveis de cada acesso, para que os mesmos possam ser utilizados adequadamente em procedimentos futuros, assim como realizado no Japão em 1911, onde praticamente todas as variedades do bicho-da-seda foram coletadas e suas características examinadas (YOKOYAMA, 1959).

No Brasil, este trabalho tem sido realizado por meio de Órgãos Oficiais e de empresas particulares, nos quais grande número de estudos foram conduzidos, objetivando comparar as várias raças do bicho-da-seda, bem como seus híbridos, quanto às suas características desejáveis (ABREU et al., 1959; ABRAMIDES e ABREU, 1960; ABREU et al. $1960 \mathrm{a} ; 1960 \mathrm{~b} ; 1962$; PAOLIERI, 1965a; 1965b; BORGONOVI \& PEDRO, 1966/1967). Atualmente a formação e distribuição dos híbridos comerciais do bicho-da-seda ficou restrita às empresas privadas que atuam no setor, seguindo um modelo integrado de produção empresa/produtor.

Assim, este trabalho teve por objetivo caracterizar oito acessos de Bombyx mori L., segundo seus atributos qualitativos e quantitativos, com vistas ao desenvolvimento de um programa de seleção e formação de um híbrido comercial do bichoda-seda, adaptado às condições ambientais e com alta produção de seda.

\section{MATERIAL E MÉTODOS}

O estudo foi desenvolvido na Estação Experimental de Zootecnia de Gália, do Instituto de Zootecnia, Secretaria de Agricultura e Abastecimento do Estado de São Paulo, no ano de 2000, quando foram analisadas oito raças do bicho-da-seda, sendo quatro de origem japonesa (B101, B102, B104, B 109) e quatro de origem chinesa (C201, C202, C203, C208), em função de caracteres biológicos de grande influência genética e relacionados direta e indiretamente com a produção de seda. Para cada raça, foram separadas aproximadamente duas mil lagartas (uma grama). Todas as raças foram criadas em ambiente controlado (temperatura média de $25,44^{\circ} \mathrm{C}$, umidade relativa do ar média de $70,68 \%$ e ventilação) e receberam o mesmo manejo, tanto alimentar (folhas do cultivar de amoreira IZ 56/4 e cinco tratos diários) quanto de criação (caixas padronizadas, aplicação de cal, tipo de bosque, etc).

No oitavo dia do quinto ínstar, foram coletadas amostras de lagartas das respectivas raças, colocadas em recipientes plásticos e levadas ao freezer. Após inativação, as mesmas foram dissecadas, sendo suas glândulas sericígenas retiradas e pesadas, determinando-se o peso unitário da glândula (GS) em gramas.

Foram avaliados o ganho de peso total para uma lagarta (GP), obtido no final do período larval, sendo expresso em gramas; a porcentagem média de mortalidade larval (MO); o número de machos (NM) e fêmeas (NF), obtido a partir da contagem em uma amostra de 30 casulos, tomada ao acaso; o número de ovos/postura (OP); a porcentagem de eclosão (EC), obtida pela contagem e transformação em porcentagem dos ovos que efetivamente eclodiram lagartas, por mariposa.

De cada raça do bicho-da-seda, foram coletados 30 casulos e seus caracteres mais importantes foram analisados, como: peso de casca sérica (CS) (após o corte dos casulos e retirada da crisálida e do espólio, as cascas foram pesadas), determinando-se o peso em gramas; peso de crisálida (PC), obtido a partir dos casulos utilizados na determinação anterior, sendo os valores expressos em gramas; casulos desclassificados (CD), obtidos pela contagem e transformação em porcentagem dos casulos não considerados como casulos de primeira, de acordo com TINOCO et al. (2000), com exceção dos casulos duplos, em uma amostra de 200 gramas de casulos, coletada aleatoriamente; teor de seda líquido (TS), obtido a partir da fórmula: \% de seda bruta $=$ peso de 30 cascas séricas/peso de 30 casulos inteiros x 100 , descontando-se $24 \%$ (perdas na fiação) do 
resultado obtém-se o valor médio; comprimento de um casulo(CC), obtido pela medição unitária de casulos, sendo os valores expressos em milímetros; largura de um casulo (LC), obtido pela medição unitária de casulos, sendo os valores expressos em milímetros.

Os dados de $\mathrm{MO}$ e CD foram transformados para $\log (\mathrm{x})$ e os de NM, NF e OP foram transformados para Raiz quadrada (x).

$\mathrm{O}$ delineamento experimental foi o inteiramente ao acaso, com quatro repetições/raça. A comparação entre as médias de raças do bicho-daseda foi realizada pelo teste de Tukey.

\section{RESULTADOS E DISCUSSÃO}

O desempenho biológico do bicho-da-seda demonstra o grau de adaptação deste inseto ao ambiente e a sua potencialidade para a produção de seda. Na tabela 1, estão apresentadas as médias de alguns caracteres biológicos de interesse para a sericicultura, como: GP, MO, NM, NF, OP e EC.

Considerando que o bicho-da-seda acumula reservas energéticas durante a fase larval para a produção de casulos e para as fases posteriores de seu ciclo biológico (crisálida, mariposa, postura), o ganho de peso (GP) no final desta fase é de grande importância. Não foram encontradas variações quanto ao GP para as raças em estudo (Tabela 1). Tal fato pode ser explicado pela capacidade de adaptação dos insetos lepidópteros às condições ambientais e ao alimento fornecido, de forma a otimizar seu crescimento e desenvolvimento (CROCOMO \& PARRA, 1985; PARRA, 1991; PAUL et al., 1992; PORTO, 2000).
A produção do casulo depende do índice de sobrevivência da lagarta que é considerado por BRASLAVSKY et al. (1997), como um dos fatores-chave em todo programa de seleção e, do qual depende a produção do casulo. Os resultados relativos a porcentagem de mortalidade (MO) não variaram, demonstrando uma grande homogeneidade entre as raças (Tabela 1). Através do cálculo da média geral da MO das raças (20,50\%), pode-se observar que os valores foram relativamente elevados, quando comparados com dados da literatura, que cita uma perda de $15 \%$ em uma criação normal (HANADA \& WATANABE, 1986), embora este dado se refira a híbridos comerciais selecionados e não a raças puras, mais susceptíveis.

Quanto ao número de machos (NM) e fêmeas (NF) observados, apenas para a primeira variável houve diferença entre as raças. As raças B102, B109, C201, C202 e C208 apresentaram maior NM somente em relação a raça B101 (Tabela 1). Em geral, as raças Japonesas apresentaram uma tendência de produzir maior número de fêmeas e as raças Chinesas um maior número de machos, estando em conformidade com os resultados observados por TOLEDO (1992).

Também para a variável número de ovos/ postura (OP), não foram observadas diferenças significativas entre as raças (Tabela 1). TOLEDO (1992), quando comparou duas raças puras do bichoda-seda (Chinesa e Japonesa), não observou variação quanto ao número de ovos por mariposa. A média geral (410 ovos/postura) está de acordo com dados da literatura, onde é afirmado que uma mariposa, em média, põe de 300 a 600 ovos (DOIRA et al., 1978; KANTARATANAKUL et al., 1987).

Tabela 1 - Médias de ganho de peso total (GP), mortalidade (MO), número de machos (NM), número de fêmeas (NF), número de ovos por postura (OP) e porcentagem de eclosão (EC), para oito raças de bicho-da-seda.

\begin{tabular}{|c|c|c|c|c|c|c|}
\hline Raças & GP (gr) & $\operatorname{MO}(\%)^{(1)}$ & $\mathrm{NM}(\mathrm{n} .)^{(2)}$ & $\mathrm{NF}(\mathrm{n} .)^{(2)}$ & $\mathrm{OP}(\mathrm{n} .)^{(2)}$ & $\mathrm{EC}(\%)$ \\
\hline B104 & $4,20 \mathrm{a}$ & $13,22(4,20) \mathrm{a}$ & $10,33(3,21) a b$ & $19,96(4,43) \mathrm{a}$ & $383,00(19,54) \mathrm{a}$ & $99,33 \mathrm{a}$ \\
\hline B102 & $3,95 \mathrm{a}$ & $23,33(3,12) \mathrm{a}$ & $13,66(3,69) \mathrm{a}$ & $18,00(4,23) \mathrm{a}$ & $389,00(19,72) \mathrm{a}$ & $97,65 \mathrm{a}$ \\
\hline B101 & $4,29 \mathrm{a}$ & $20,66(2,42) \mathrm{a}$ & $6,00(2,42) b$ & $24,00(4,90) \mathrm{a}$ & $426,66(20,58 \mathrm{a}$ & $75,58 \mathrm{~b}$ \\
\hline B109 & $4,22 \mathrm{a}$ & $14,29(2,49) \mathrm{a}$ & $10,33(3,20) \mathrm{a}$ & $19,66(4,43) \mathrm{a}$ & $412,00(20,30) \mathrm{a}$ & $98,30 \mathrm{a}$ \\
\hline C201 & $3,95 \mathrm{a}$ & $25,14(3,22) a$ & $13,33(3,62) \mathrm{a}$ & $16,66(4,06) \mathrm{a}$ & $412,00(20,28) a$ & $91,63 \mathrm{a}$ \\
\hline C202 & $4,13 \mathrm{a}$ & $23,33(3,05) \mathrm{a}$ & $13,66(3,68) \mathrm{a}$ & $16,33(4,03) \mathrm{a}$ & $407,66(20,18) a$ & $96,24 \mathrm{a}$ \\
\hline C203 & $3,99 \mathrm{a}$ & $14,00(2,40) \mathrm{a}$ & $10,66(3,25) \mathrm{ab}$ & $19,33(4,39) \mathrm{a}$ & $359,00(18,93)$ a & $87,73 \mathrm{ab}$ \\
\hline C208 & $3,77 \mathrm{a}$ & $30,00(3,37) \mathrm{a}$ & $12,33(3,51) \mathrm{a}$ & $17,66(4,20) \mathrm{a}$ & $489,00(22,09) \mathrm{a}$ & $96,15 \mathrm{a}$ \\
\hline Média Geral & 4,06 & $20,50(2,90)$ & $11,29(3,32)$ & $18,91(4,33)$ & $409,79(20,20)$ & 92,83 \\
\hline $\mathrm{CV} \%$ & 4,70 & $17,51^{(3)}$ & $11,20^{(3)}$ & $7,58^{(3)}$ & $5,91^{(3)}$ & $1,49^{(3)}$ \\
\hline
\end{tabular}

Médias seguidas de letras distintas, nas colunas, diferem entre si pelo teste de Tukey $(\mathrm{P}<0,05)$;

${ }^{(1)}$ Médias entre parêntesis são médias transformadas para $\log (\mathrm{x})$;

(2) Médias entre parêntesis são médias transformadas para Raiz quadrada (x);

${ }^{(3)} \mathrm{CV} \%$ transformados para $\log (\mathrm{x})$ ou Raiz quadrada (x). 
A porcentagem de eclosão (EC) é um importante fator pelo qual se avalia a resistência do bicho-da-seda às condições adversas do ambiente $\mathrm{e}$ às doenças, bem como sua prolificidade. AEC da raça $\mathrm{B} 101$ foi inferior às demais, não diferindo apenas em relação à raça C203. Considerando como ideal uma eclosão acima de 90\% (ABREU \& ABRAMIDES, 1974), na média geral $(92,83 \%)$ as raças apresentaram bom desempenho, com exceção para as raças B101 e C203 (Tabela 1).

O casulo do bicho-da-seda e conseqüentemente o fio de seda obtido, são os objetivos básicos da exploração Sericícola, portanto suas características desempenham importante fator na seleção de raças especializadas. Na tabela 2 estão apresentados alguns caracteres relacionados à produção de seda, como: GS, CS, PC, TS, CD, CC e LC, para as oito raças em estudo.

Os caracteres peso da glândula sericígena (GS), peso de casca sérica (CS) e peso de crisálida (PC) determinam valores para a produção de seda, já o teor de seda líquido (TS) é um índice para avaliar comercialmente a produção.

Apenas a raça C201 apresentou valores inferiores para GS (Tabela 2). Transformando estes pesos em porcentagem do peso total da lagarta, os seguintes valores podem ser observados: $32 \%, 32 \%$, $36 \%, 35 \%, 32 \%, 38 \%, 32 \%$ e $34 \%$ para as raças B 104 , B102, B101, B109, C201, C202, C203 e C208, respectivamente. Nota-se uma boa proporção quanto ao peso da glândula sericígena em relação ao peso corporal da lagarta para a raça Chinesa C202 (38\%), demonstrando uma boa capacidade para produção de seda. Estes resultados estão de acordo com FONSECA $\&$ FONSECA (1988) que determinaram porcentagens entre 20 a $40 \%$.

Quanto ao peso da casca sérica (CS), duas raças de origem Japonesa (B101 e B104) e três raças de origem Chinesa (C202, C203 e C208) apresentaram valores médios superiores, sendo que apenas a raça B 104 apresentou um PC superior, denotando importantes características tanto produtivas quanto biológicas. Os piores resultados quanto ao CS e PC foram observados para a raça C201. Para a variável TS, em geral, as raças chinesas apresentaram teores mais elevados, destacando-se a raça C202 (Tabela 2).

A presença de casulos de segunda, aqui denominados de casulos desclassificados (CD), é característica indesejável que pode ter origem genética. As raças Japonesas (B104 e B109) apresentaram os menores valores para $\mathrm{CD}$. A raça $\mathrm{C} 201$ foi a que apresentou um dos valores mais elevados para CD (Tabela 2).

As características externas do casulo, relacionadas a sua forma, estão normalmente ligadas à raça do bicho-da-seda (BORGONOVI,1955). As raças Japonesas, em geral, apresentaram casulos de maior comprimento (CC), destacando-se as raças B101 e B104. A raça $\mathrm{C} 201$ produziu casulos de menor comprimento. Quanto à largura (LC), as raças Chinesas produziram casulos com valores superiores, destacando-se as raças C203 e C208 (Tabela 2). Estes resultados estão de acordo com OKINO (1982), que afirmou que as raças de origem Japonesa produzem casulos médios e de forma alongada, enquanto as raças Chinesas produzem casulos de forma esférica a oval.

Tabela 2 - Médias de peso unitário da glândula sericígena (GS), peso de 30 cascas séricas (CS), peso de 30 crisálidas (PC), teor de seda líquido (TS), casulos desclassificados (CD), comprimento de um casulo (CC) e largura de um casulo (LC), para oito raças do bicho-da-seda.

\begin{tabular}{lccccccc}
\hline Raças & GS $(\mathrm{gr})$ & CS $(\mathrm{grs})$ & PC $(\mathrm{grs})$ & TS $(\%)$ & CD $(\%)^{(1)}$ & CC $(\mathrm{mm})$ & $\mathrm{LC}(\mathrm{mm})$ \\
\hline B104 & $0,959 \mathrm{a}$ & $9,275 \mathrm{a}$ & $36,125 \mathrm{a}$ & $15,82 \mathrm{~b}$ & $9,89(2,27) \mathrm{b}$ & $32,60 \mathrm{a}$ & $15,55 \mathrm{~d}$ \\
B102 & $0,971 \mathrm{a}$ & $7,150 \mathrm{~b}$ & $29,450 \mathrm{de}$ & $15,07 \mathrm{~b}$ & $12,01(2,43 \mathrm{ab}$ & $29,49 \mathrm{bc}$ & $14,21 \mathrm{f}$ \\
B101 & $1,072 \mathrm{a}$ & $8,600 \mathrm{a}$ & $33,55 \mathrm{~b}$ & $15,86 \mathrm{~b}$ & $13,53(2,56) \mathrm{ab}$ & $31,73 \mathrm{a}$ & $14,98 \mathrm{de}$ \\
B109 & $0,924 \mathrm{a}$ & $7,575 \mathrm{~b}$ & $28,200 \mathrm{e}$ & $16,22 \mathrm{~b}$ & $9,12(2,04) \mathrm{b}$ & $30,14 \mathrm{~b}$ & $14,62 \mathrm{ef}$ \\
C201 & $0,648 \mathrm{~b}$ & $7,125 \mathrm{~b}$ & $25,650 \mathrm{f}$ & $16,66 \mathrm{ab}$ & $23,37(3,15) \mathrm{a}$ & $26,50 \mathrm{e}$ & $17,41 \mathrm{c}$ \\
C202 & $1,006 \mathrm{a}$ & $9,350 \mathrm{a}$ & $30,400 \mathrm{~cd}$ & $18,10 \mathrm{a}$ & $14,63(2,68 \mathrm{ab}$ & $28,95 \mathrm{~cd}$ & $18,32 \mathrm{~b}$ \\
C203 & $1,036 \mathrm{a}$ & $9,175 \mathrm{a}$ & $33,350 \mathrm{~b}$ & $16,55 \mathrm{ab}$ & $16,75(2,80 \mathrm{ab}$ & $28,09 \mathrm{~d}$ & $19,42 \mathrm{a}$ \\
C208 & $0,924 \mathrm{a}$ & $8,875 \mathrm{a}$ & $31,675 \mathrm{bc}$ & $16,70 \mathrm{ab}$ & $10,87(2,35) \mathrm{ab}$ & $28,73 \mathrm{~cd}$ & $19,46 \mathrm{a}$ \\
Média Geral & 0,943 & 9,391 & 31,050 & 16,37 & $13,77(2,54)$ & 29,53 & 16,75 \\
CV\% & 10,18 & 8,39 & 2,64 & 4,58 & 14,38 & 1,60 & 1,96 \\
\hline
\end{tabular}

Médias seguidas de letras distintas, nas colunas, diferem entre si pelo teste de Tukey $(\mathrm{P}<0,05)$;

${ }^{(1)}$ Médias entre parêntesis são médias transformadas para $\log (\mathrm{x})$;

${ }^{(2)} \mathrm{CV} \%$ transformado para $\log (\mathrm{x})$. 
Pode-se observar ainda uma relação entre o CC, PC e TS. Os casulos produzidos por lagartas da raça $\mathrm{C} 201$ apresentaram menor $\mathrm{CC}$, menor $\mathrm{PC}$ e um TS que não diferiu das demais, o que pode ser explicado por BORGONOVI (1955), quando afirmou que os casulos pequenos, em geral, apresentam maior riqueza em seda, em virtude do menor peso das suas crisálidas, uma vez que o TS é obtido através de uma proporção (CS/Peso de casulo inteiro).

As informações levantadas neste estudo permitem uma avaliação preliminar do material genético disponível, de forma a direcionar um programa de seleção e formação de um híbrido comercial do bichoda-seda.

\section{CONCLUSÕES}

As raças Japonesas e Chinesas apresentaram grande homogeneidade quanto ao ganho de peso, mortalidade, número de fêmeas e número de ovos/postura.

A raça B101 apresentou um menor número de machos e uma menor porcentagem de eclosão. Entre as raças de origem Japonesa, a B104 apresentou as melhores características de produção de casulo. No geral, a raça de origem Chinesa C202 apresentou as melhores características de produção de casulo e a raça de origem Chinesa C201, as piores características.

\section{REFERÊNCIASBIBLIOGRÁFICAS}

ABRAMIDES, P.; ABREU, O.C. Estudo de híbridos na entressafra. Campinas, Boletim Técnico de Sericicultura Campinas, n.27, 15p, 1960

ABREU, O.C.; ABRAMIDES, P. Técnica de criação do bichoda-seda Bombyx mori L. Boletim Técnico de Sericicultura, Campinas, n.3, 28p, 1974.

ABREU, O.C. et al. Ensaio de híbridos do bicho-da-seda. Boletim Técnico de Sericicultura, Campinas, n.28, 18p, 1959.

ABREU, O.C. et al. Ensaios com raças puras do bicho-daseda. Boletim Técnico de Sericicultura, Campinas, n.22, $18 \mathrm{p}, 1960 \mathrm{a}$

ABREU, O.C. et al. Ensaios de raças puras do bicho-da-seda Boletim Técnico de Sericicultura, Campinas, n.30, 22p, 1960 b.

ABREU, O.C. et al. Ensaios com raças de bicho-da-seda de procedências chinesa, japonesa e européia. Boletim Técnico de Sericicultura, Campinas, n.15, 20p, 1962

BORGONOVI, A. Técnica da fiação e da classificação da seda. Boletim Técnico de Sericicultura, Campinas, n.8, 53p, 1955.
BORGONOVI, A.; PEDRO, J.R. Influência das raças sobre a pureza da seda "grege". Boletim Técnico de Sericicultura, Campinas, n.48, 13p, 1966.

BORGONOVI, A.; PEDRO, J.R. Características de casulos provenientes de raças do bicho-da-seda Bomby $x$ mori introduzidos ultimamente na sericicultura. Boletim Técnico de Sericicultura, Campinas, n.44, 11p, 1967.

BRASLAVSKY, M.E. et al. Working out base silkworm selection programs in Ukraine. In: CONGRESSO DA COMISSÃO SERICÍCOLA INTERNACIONAL, 17., 1997, Londrina. Anais... Londrina : Governo do Estado do Paraná, 1997. Seção 2, p.147.

CROCOMO, W.B.; PARRA, J.R.P. Consumo e utilização de milho, trigo e sorgo por Spodoptera frugiperda (J.E.Smith, 1797)(Lepidóptera: noctuidae). Revista Brasileira de Entomologia.,v.29, n.2, p.225-260, 1985.

DOIRA, H. et al. The silkworm, an important laboratory tool. Tokyo : Kodansha, 1978. 307p.

FONSECA, T.C.; FONSECA, A.S. Cultura da amoreira e criação do bicho-da-seda. São Paulo : Nobel, 1988. 246p.

HANADA, Y.; WATANABE, J.K. Manual de criação do bicho-da-seda. Curitiba : COCAMAR, 1986. 224p.

KANTARATANAKUL, S. et al. Heterosis in F1 hybrid between polyvoltine and bivoltine silkworm (Bombyx mori L.). Sericologia, v.27, n.3, p.373-380. 1987

KRISHNASWAMI, S. et al. Sericulture manual 2silkworm rearing. Rome : Food and Agriculture Organization of the United Nations, 1979. 131p.

OKINO, I. Manual de sericicultura. Bauru : ni., 1982. $80 \mathrm{p}$.

PANG-CHUAN, W.; DA-CHUANG, C. Silkworm rearing. Rome : Food and Agriculture Organization of the United Nations, 1992. 83p.

PAOLIERI, L. Estudos experimentais comparativos de raças de bicho-da-seda (Bombyx mori L.). Boletim Técnico de Sericicultura, Campinas, n.45, 56p, 1965a.

PAOLIERI, L. Estudos experimentais de raças e híbridos de bicho-da-seda. Boletim Técnico de Sericicultura Campinas, n.52, 47p, 1965 b.

PARRA, J.R.P. Consumo e utilização de alimento por insetos. In: PANIZZI, A.R.; PARRA, J.R.P. Ecologia nutricional de insetos e suas implicações no manejo de pragas. São Paulo : Nobel, 1991. p.9-66.

PAUL, D.C.; SUBBARAO, G.; DEB, D.C. Impact of dietary moisture on nutritional indices and growth of Bombyx mori L. and concommitant larval duration. Journal Insect Physiology, v.38, n.3, p.229-245, 1992.

PORTO, A.J. Efeito da idade de corte de dois cultivares de amoreira no desempenho e características do casulo do bicho-da-seda (Bombyx mori L.). 2000 . $81 \mathrm{f}$ Dissertação (Mestrado em Zootecnia) - Faculdade de Medicina Veterinária e Zootecnia, Universidade Estadual Paulista. 
RAJU, P.J.; KRISHNAMURTHY, N.B. Breeding of two bivoltines, MG511 and MG512, of silkworm, Bombyx mori L., for higher viability and silk productivity. Sericologia, v.33, n.4, p.577-587. 1993.

RAVINDRA SINGH, J. et al. Heterosis analysis in the silkworm, Bombyx mori L. Seriologia, v.30, n.3, p.293-300. 1990.

TINOCO, S.T.J. et al. Manual de sericicultura. Campinas : CATI, 2000. 75p. (Manual técnico 75).
TOLEDO, J.O. Análise do desempenho biológico e das características tecnológicas do casulo de raças puras híbridos de bicho-da-seda (Bombyx mori L.). 1992. 98f. Dissertação (Mestrado em Zootecnia) Faculdade de Ciências Agrárias e Veterinárias, Universidade Estadual Paulista.

YOKOYAMA, T. Silkworm genetics illustrated. Tóquio : Japan, Society for the Promotions of Science, 1959. $185 \mathrm{p}$. 